\title{
Intergenerational equity and an explicit construction of welfare criteria
}

\author{
Toyotaka Sakai ${ }^{* \dagger}$
}

August 19, 2008

\begin{abstract}
Ranking infinite utility streams includes many impossibility results, most involving certain Pareto, anonymity, or continuity requirements. We introduce the concept of the future agreement extension, a method that explicitly extends orderings on finite time horizons to an infinite time horizon. The future agreement extension of given orderings is quasi-transitive, complete, and pairwisely continuous. Furthermore, it is a subrelation of any other pairwisely continuous extension of the orderings. In case of anonymous and strongly Paretian orderings, their future agreement extension is variable step anonymous and strongly Paretian. Characterizations of the future agreement extensions of the utilitarian and leximin orderings are obtained as applications.
\end{abstract}

Key words: Future agreement extension, Variable step anonymity, Intergenerational equity, Infinite generations, Diamond's impossibility theorem.

JEL Codes: D71, D63, D 60.

*Faculty of Economics and IGSSS, Yokohama National University, Yokohama 240-8501, Japan; toyotaka@ynu.ac.jp; http://www.geocities.jp/toyotaka_sakai/

${ }^{\dagger}$ I thank Chiaki Hara, Tomoichi Shinotsuka, Kotaro Suzumura, and Koichi Tadenuma for their helpful comments on an earlier version at the Workshop on Intergenerational Equity at Hitotsubashi University, February 2008. The financial support by KAKENHI (19310031) is gratefully acknowledged. 


\section{Introduction}

Dynamic economic environments are often modelled to incorporate an infinite number of generations. However, ethically evaluating intergenerational welfare in such models is non-trivial because of the difficulty of dealing with infinity. Indeed, since the seminal work by Diamond (1965), various impossibility results have been obtained for the construction of intergenerational welfare criteria satisfying basic normative requirements. This is in contrast with the existence of many reasonable welfare orderings for finite populations, and as a resolution, we shall consider extending such welfare orderings to an infinite time horizon.

A sequence of finite generation orderings is $R=\left\{R_{t}\right\}_{t \in \mathbb{N}}$, where each $R_{t}$ is a welfare ordering at period $t$ that ranks $t$-dimensional utility streams. Examples are the sequences of the utilitarian and leximin orderings. Our idea of constructing an intergenerational welfare criterion based on such a sequence is very simple. When two infinite utility streams $x=\left(x_{1}, x_{2}, \ldots\right)$ and $y=\left(y_{1}, y_{2}, \ldots\right)$ are given, $x$ is ranked higher than $y$, if and only if there exists a time period $s$ such that all future periods $t \geq s$ agree with the normative judgement at period $s$ that ranks $x$ higher than $y$, where each period $t$ is concerned with the $t$-period histories given by $x$ and $y$, that is,

$$
\left(x_{1}, x_{2}, \ldots, x_{t}\right) P_{t}\left(y_{1}, y_{2}, \ldots, y_{t}\right) \quad \forall t \geq s
$$

Otherwise, $x$ and $y$ are ranked indifferently. The binary relation so constructed is called the future agreement extension of $R$. This method is quite useful as it applies to any arbitrary sequence of finite generation orderings and considerable knowledge exists about orderings defined on finite dimensions in social choice theory (see, d'Aspremont and Gevers, 2002, for a survey).

Needless to say, all impossibility results in this literature apply to the future agreement extension. For example, the non-existence of binary relations satisfying weak Pareto, finite anonymity, and product continuity (Diamond, 1965) implies that the future agreement extension of any sequence cannot satisfy the three requirements simultaneously. However, a great advantage of the future agreement extension is that it generates a complete binary relation satisfying all standard requirements at some good level. Particularly, whenever a sequence consists of anonymous orderings, its future agreement extension satisfies a strong impartiality requirement of variable step anonymity (Lauwers, 1997b). Furthermore, it is strongly Paretian if the orderings are strongly Paretian. On transitivity conditions, the future agreement extension achieves quasi-transitivity and finite transitivity but not transitivity. This impossibility is, however, an inevitable consequence of the fact that no binary relation can be transitive, weakly Paretian, and variable step anonymous (Lauwers, 1997b; Fleurbaey and 
Michel, 2003).

For every sequence of finite generation orderings, its future agreement extension satisfies a continuity condition based on the pairwise convergence of infinite utility streams. This condition is weaker than the product, Campbell, and Mackey continuities. Since these three continuity conditions are incompatible even with finite anonymity and weak Pareto (Diamond, 1965; Campbell, 1985; Shinotsuka, 1998), our pairwise continuity can be seen as an appropriate relaxation of these continuity conditions so as to be compatible with even stronger notions of variable step anonymity and strong Pareto.

We obtain several characterization results. The future agreement extension of a given sequence is a subrelation of any pairwisely continuous extension of the sequence, and it is the unique extension under an additional requirement of Asheim and Tungodden's (2004) continuity condition. We also show that a version of d'Aspremont's extension (2007) is a superrelation of any pairwisely continuous extension under a few standard normative requirements. These results are applied to obtain characterizations of the extensions of the sequences of the utilitarian and leximin orderings.

This paper is organized as follows: Section 2 offers definitions. Section 3 introduces the future agreement extension and investigates its properties. Section 4 provides main results. Section 5 gives concluding comments.

\section{Definitions}

\section{$2.1 \quad$ Basic notion}

Let $\mathbb{N} \equiv\{1,2, \ldots\}$ be the set of infinite generations. A unit interval $X \equiv[0,1]$ is called a domain, which is the set of possible utility levels of each generation. A utility stream is a vector $x \equiv\left(x_{1}, x_{2}, \ldots\right) \in X^{\infty}$, where each $x_{t}$ denotes the utility level of the generation $t$. Given $x, y \in X^{\infty}$ and $t \in \mathbb{N}$, we define their " $t$-head" and " $t+1$-tail" by

$$
\begin{aligned}
x(t) & \equiv\left(x_{1}, x_{2}, \ldots, x_{t}\right) \in X^{t}, \\
y[t+1] & \equiv\left(y_{t+1}, y_{t+2}, \ldots\right) \in X^{\infty},
\end{aligned}
$$

respectively, and denote their combination stream by

$$
(x(t), y[t+1]) \equiv\left(x_{1}, x_{2}, \ldots, x_{t}, y_{t+1}, y_{t+2}, \ldots\right) \in X^{\infty}
$$

We also define, for $t, s \in \mathbb{N}$ with $t<s$,

$$
(x(t), y[t+1, s]) \equiv\left(x_{1}, x_{2}, \ldots, x_{t}, y_{t+1}, y_{t+2}, \ldots, y_{s}\right) \in X^{s}
$$


A binary relation is a subset $\succsim \subseteq X^{\infty} \times X^{\infty}$, and its asymmetric and symmetric parts are defined by $\succ \equiv\{(x, y) \in \succsim:(y, x) \notin \succsim\}$ and $\sim \equiv\{(x, y) \in \succsim:(y, x) \in \succsim\}$, respectively. We often write $x \succsim y$ instead of $(x, y) \in \succsim$, and the same notational rule applies to $\succ$ and $\sim$. Given $x, y \in X^{\infty}$, vector inequalities are defined by

$$
\begin{aligned}
& x \geqq y \Longleftrightarrow\left[x_{t} \geq y_{t} \quad \forall t \in \mathbb{N}\right], \\
& x \geq y \Longleftrightarrow\left[x_{t} \geq y_{t} \quad \forall t \in \mathbb{N} \text { and } x_{s}>y_{s} \quad \exists s \in \mathbb{N}\right], \\
& x>y \Longleftrightarrow\left[x_{t}>y_{t} \quad \forall t \in \mathbb{N}\right] .
\end{aligned}
$$

We define various properties of binary relations:

Order properties A binary relation $\succsim$ is transitive if $x \succsim y$ and $y \succsim z$ imply $x \succsim z$ for every $x, y, z \in X^{\infty}$, is quasi-transitive if $x \succ y$ and $y \succ z$ imply $x \succ z$ for every $x, y, z \in X^{\infty}$, is finitely transitive if $x \succ y$ and $y \succ z$ imply $x \succ z$ for every $x, y, z \in X^{\infty}$ such that $x[t+1]=y[t+1]=z[t+1]$ for some $t \in \mathbb{N}$, and is complete if $x \succsim y$ or $y \succsim x$ for every $x, y \in X^{\infty}$.

Pareto properties A binary relation $\succsim$ is weakly Paretian if $x \succ y$ for every $x, y \in X$ with $x>y$, and is strongly Paretian if $x \succ y$ for every $x, y \in X$ with $x \geq y$.

Anonymity properties A permutation is a bijection $\pi: \mathbb{N} \rightarrow \mathbb{N}$. The set of all permutations is denoted by $\bar{\Pi}$. Given $\Pi \subseteq \bar{\Pi}$, a binary relation $\succsim$ on $X$ satisfies I-anonymity if $\pi(x) \equiv\left(x_{\pi(t)}\right)_{t \in \mathbb{N}} \sim x$ for every $x \in X$ and every $\pi \in \Pi$. Interesting classes of permutations are as follows:

- A permutation $\pi \in \bar{\Pi}$ is a finite permutation if there exists an integer $s>0$ such that for each $t \geq s, \pi(t)=t$. Let $\Pi_{1}$ be the set of finite permutations. $\Pi_{1}$-anonymity is called finite anonymity. Finite anonymity has been the most studied in the literature since Diamond (1965). It is known that there exists a binary relation satisfying transitivity, completeness, strong Pareto, and finite anonymity (Svensson, 1980). ${ }^{1}$

- A permutation $\pi \in \bar{\Pi}$ is a fixed step permutation if there exists a "length" integer $s>0$ such that for each "step" integer $n>0$,

$$
\{1,2, \ldots, n s\}=\{\pi(1), \pi(2), \ldots, \pi(n s)\}
$$

Let $\Pi_{2}$ be the set of fixed step permutations. $\Pi_{2}$-anonymity is called fixed step anonymity. This axiom is introduced by Lauwers (1997b). Likewise

\footnotetext{
${ }^{1}$ Studies that focus on finitely anonymous binary relations include Diamond (1965), Svensson (1980), Campbell (1985), Lauwers (1997a,c), Shinotsuka (1998), Fleurbaey and Michel (2003), and Sakai (2003a,b).
} 
as finite anonymity, there exists a binary relation satisfying transitivity, completeness, strong Pareto, and fixed step anonymity (Lauwers, 1997b; Fleurbaey and Michel, 2003). ${ }^{2}$

- A permutation $\pi \in \bar{\Pi}$ is a variable step permutation if there exists a sequence of integers $s_{1}, s_{2}, s_{3}, \ldots$ with $0<s_{1}<s_{2}<s_{3}<\cdots$ such that for each member $s_{k}$ of the sequence,

$$
\left\{1,2, \ldots, s_{k}\right\}=\left\{\pi(1), \pi(2), \ldots, \pi\left(s_{k}\right)\right\}
$$

Let $\Pi_{3}$ be the set of variable step permutations. $\Pi_{3}$-anonymity is called variable step anonymity. This axiom is sketched by Lauwers (1997b) and is studied by Fleurbaey and Michel (2003), however, not much effort has been taken to understand its implications. Fleurbaey and Michel (2003) simultaneously demonstrate the existence of a binary relation satisfying quasitransitivity, completeness, strong Pareto, and variable step anonymity, with the non-existence of a binary relation satisfying transitivity, weak Pareto, and variable step anonymity.

Moreover, $\bar{\Pi}$-anonymity, the strongest anonymity condition, is called infinite anonymity. ${ }^{3}$ There exists no binary relation satisfying strong Pareto and infinite anonymity (e.g., van Liedekerke and Lauwers, 1997). It is obvious that $\Pi_{1} \subsetneq$ $\Pi_{2} \subsetneq \Pi_{3} \subsetneq \bar{\Pi}$; however, the choice of permutations is controversial.

\subsection{Extensions of finite generation orderings}

Given $t \in \mathbb{N}$, a binary relation on $X^{t}$ is denoted by $R_{t}$, where its asymmetric and symmetric parts are denoted by $P_{t}$ and $I_{t}$, respectively. Order properties and Pareto properties of $R_{t}$ are defined by the same way as $\succsim$ on $X^{\infty}$. When there are only finite generations, there is a unique definition of anonymity, because the choice of the class of permutations is not a problem. That is, $R_{t}$ on $X^{t}$ is anonymous if $x(t) I_{t}\left(x_{\pi(s)}\right)_{s=1}^{t}$ for every $x(t) \in X^{t}$ and every permutation $\pi$ on $\{1,2, \ldots, t\}$. A binary relation $R_{t}$ on $X^{t}$ is an ordering if it is transitive and complete.

The following are two famous examples of strongly Paretian and anonymous orderings on $X^{t}$ :

\footnotetext{
${ }^{2}$ This desirability has attracted the attention of recent researches (e.g., Fleurbaey and Michel, 2003; Banerjee, 2006; Mitra and Basu, 2007; Kamaga and Kojima, 2007a,b; Kamaga, 2008).

${ }^{3}$ A further stronger axiom, called "reinforcement", can be found in Chambers (2008). This axiom states invariance of ranking under any arbitrary permutation and concatenation of utility streams.
} 
Utilitarian ordering, $R_{t}^{U}$ : For all $x(t), y(t) \in X^{t}$,

$$
x(t) R_{t}^{U} y(t) \Longleftrightarrow \sum_{s=1}^{t} x_{s} \geq \sum_{s=1}^{t} y_{s} .
$$

Leximin ordering, $R_{t}^{L}$ : For all $x(t), y(t) \in X^{t}$,

$$
\begin{aligned}
& x(t) I_{t}^{L} y(t) \Longleftrightarrow x(t)=y(t), \\
& x(t) P_{t}^{L} y(t) \Longleftrightarrow \exists s \leq t,\left[\overline{x(t)}_{s}>\overline{y(t)}_{s} \text { and } \overline{x(t)}_{s^{\prime}}=\overline{y(t)}_{s^{\prime}} \forall s^{\prime}<s\right],
\end{aligned}
$$

where $\overline{x(t)}$ and $\overline{y(t)}$ are $t$-dimensional utility streams obtained by permuting $x(t)$ and $y(t)$ in ascending order, respectively.

A sequence of finite generation orderings is a sequence $R \equiv\left\{R_{t}\right\}_{t \in \mathbb{N}}$ where $R_{t}$ is an ordering on $X^{t}$ for every $t \in \mathbb{N}$. Such a sequence $R$ is correlated if for every $x, y, z \in X^{\infty}$ and every $t \in \mathbb{N}$,

$$
x(t) R_{t} y(t) \Longleftrightarrow\left(x(t), z_{t+1}\right) R_{t+1}\left(y(t), z_{t+1}\right) .
$$

The sequences of the utilitarian and leximin orderings, $R^{U} \equiv\left\{R_{t}^{U}\right\}_{t \in \mathbb{N}}$ and $R^{L} \equiv$ $\left\{R_{t}^{L}\right\}_{t \in \mathbb{N}}$, are examples of correlated sequences of finite generation orderings.

While the existence of desirable binary relations on an infinite dimensional space is a very non-trivial question, welfare economics has provided various desirable orderings, such as $R_{t}^{U}$ or $R_{t}^{L}$, on finite dimensional spaces. Therefore, as an efficient use of accumulated knowledge, we shall somehow obtain binary relations on $X^{\infty}$ by extending a sequence of finite generation orderings $R=\left\{R_{t}\right\}_{t \in \mathbb{N}}$ to $X^{\infty}$. For this, we need formal definitions of "extension": A binary relation $\succsim$ on $X^{\infty}$ is a weak extension of $R=\left\{R_{t}\right\}_{t \in \mathbb{N}}$ if for every $x, y, z \in X^{\infty}$ and every $t \in \mathbb{N}$,

$$
x(t) R_{t} y(t) \Longrightarrow(x(t), z[t+1]) \succsim(y(t), z[t+1]),
$$

and is a strict extension of $R=\left\{R_{t}\right\}_{t \in \mathbb{N}}$ if for every $x, y, z \in X^{\infty}$ and every $t \in \mathbb{N}$,

$$
x(t) R_{t} y(t) \Longleftrightarrow(x(t), z[t+1]) \succsim(y(t), z[t+1]) .
$$

d'Aspremont (2007, Section 3) is an earlier work on extensions of a sequence of finite generation orderings. He considers the concept of a "proliferating" sequence instead of our correlated sequence, and defines the concept of "extension" with adding a flavor of strong Pareto. However, the definition of proliferation involves a consistency requirement to extended binary relations, so that checking the satisfaction is not trivial. Contrastingly, our simple definition of correlation does not involve any condition on extension, so it is purely defined as a condition on sequences. Furthermore, our weak and strict extensions are defined independent of strong Pareto, implying that non-Paretian welfare criteria are not excluded from the scope of our analysis. 


\section{Future agreement extension}

\subsection{Definition and basic properties}

We propose an extension called the future agreement extension, which is based on the idea of respecting evaluation over histories by each generation. The idea of constructing the future agreement extension $\succsim_{R}^{a}$ of $R$ is as follows: Pick any $x, y \in X^{\infty}$, and assume $x(t) P_{t} y(y)$ for some $t \in \mathbb{N}$. This means that history $x(t)$ is normatively better than history $y(t)$ when they are evaluated at period $t$. If this normative evaluation is unanimously agreed by all future generations, i.e., $x(s) P_{s} y(s)$ for all $s \geq t$, then we set $x \succ_{R}^{a} y$ :

$$
x \succ_{R}^{a} y \Longleftrightarrow \exists t \in \mathbb{N}, \forall s \geq t, x(s) P_{s} y(s) .
$$

Consider the case that some future generation $s>t$ does not agree the evaluation at $t$. Then we consider the evaluation at $t$ as imprecise, since it is not approved by a future generation whose informational bases $x(s), y(s)$ are richer than $x(t), y(t)$. When such a reversal occurs at each and every time period, we set the two steams to be indifferent:

$$
x \sim_{R}^{a} y \Longleftrightarrow \text { neither } x \succ_{R}^{a} y \text { nor } y \succ_{R}^{a} x .
$$

More formally, the future agreement extension of $R$ is defined to be the binary relation $\succsim_{R}^{a}$ on $X^{\infty}$ such that

$$
x \succsim_{R}^{a} y \Longleftrightarrow \forall t \in \mathbb{N}, \exists s \geq t, x(s) R_{s} y(s), \quad \forall x, y \in X^{\infty} .
$$

It is obvious that (1) and (2) together are equivalent to (3).

One may consider that the future agreement extension admits large indifference classes and hence is not decisive, but our Theorem 1 will show that this extension is still the best in view of decisiveness under some conditions. We are not saying that the future agreement extension is ideal, but saying that this can be a possible compromise given indeed many impossibility results in the literature. Its performance will be tested in this and the next sections.

The explicit formula of the future agreement extension is quite rare for welfare criteria with regard to infinite generations. As pointed out by Fleurbaey and Michel (2003, p.794), despite the presence of some well-behaved complete binary relations in the literature, their existence are proved using Szpilrajn's lemma (e.g., Svensson, 1980; Fleurbaey and Michel, 2003; Sakai, 2003a), which is a very non-constructive method. ${ }^{4}$

\footnotetext{
${ }^{4}$ This non-constructiveness is formally proved by Zame (2007) and Lauwers (2007) in terms of measurability or the axiom of choice.
} 
Since any welfare criterion is supposed to be used for welfare evaluation, invisibility of its explicit formula is a serious drawback in understanding how its value judgement is given. Our future agreement extension overcomes this problem. Note also that

$$
x \succsim_{R}^{a} y \Longleftrightarrow\left|\left\{t \in \mathbb{N}: x(t) R_{t} y(t)\right\}\right| \geq\left|\left\{t \in \mathbb{N}: y(t) R_{t} x(t)\right\}\right| \forall x, y \in X^{\infty}
$$

In view of (4), the future agreement extension can be seen as a plurality rule when the infinite generations are voters and each generation evaluates their histories by their welfare criterion.

The next lemma ensures that the future agreement extension of any correlated sequence is in fact a strict extension.

Lemma 1. Let $R=\left\{R_{t}\right\}_{t \in \mathbb{N}}$ be any correlated sequence of finite generation orderings. Then $\succsim_{R}^{a}$ is a strict extension of $R$.

Proof. Let $x, y, z \in X^{\infty}$ and $t \in \mathbb{N}$.

Assume $x(t) R_{t} y(t)$. Then by correlation of $R$,

$$
(x(t), z[t+1, s]) R_{s}(y(t), z[t+1, s]) \quad \forall s \geq t .
$$

Hence, by definition of $\succsim_{R}^{a}$,

$$
(x(t), z[t+1]) \succsim_{R}^{a}(y(t), z[t+1]) .
$$

Assume next $(x(t), z[t+1]) \succsim_{R}^{a}(y(t), z[t+1])$. By definition of $\succsim_{R}^{a}$, there exists $s \geq t$ such that

$$
(x(t), z[t+1, s]) R_{s}(y(t), z[t+1, s]) .
$$

If $y(t) P_{t} x(t)$, then correlation of $R$ implies

$$
(y(t), z[t+1, s]) P_{s}(x(t), z[t+1, s]) \forall s \geq t
$$

a contradiction to $(5)$.

The next lemma shows that axioms satisfied by finite generation orderings can be lifted to infinite horizon by the future agreement extension. ${ }^{5}$

\footnotetext{
${ }^{5}$ Although many other axioms can be lifted, we here deal only with the ones that are most relevant to the present study. An important example of such axioms is the Pigou-Dalton transfer principle. This axiom and its variants can be found in Fleurbaey and Michel (2001), Sakai (2003a, 2006), Bossert, Sprumont, and Suzumura (2007), and Hara, Shinotsuka, Suzumura, and Xu (2008).
} 
Lemma 2. Let $R=\left\{R_{t}\right\}_{t \in \mathbb{N}}$ be any sequence of finite generation orderings that satisfy strong Pareto and anonymity. Then $\succsim_{R}^{a}$ satisfies quasi-transitivity, completeness, strong Pareto, and variable step anonymity. If $R$ is correlated, then $\succsim_{R}^{a}$ also satisfies finite transitivity.

Proof. Let us prove quasi-transitivity. Assume that $x \succ_{R}^{a} y$ and $y \succ_{R}^{a} z$. Then there exist $t^{\prime}, t^{\prime \prime} \in \mathbb{N}$ for which

$$
\begin{aligned}
& x(t) P_{t} y(t) \quad \forall t \geq t^{\prime} \\
& y(t) P_{t} z(t) \quad \forall t \geq t^{\prime \prime}
\end{aligned}
$$

so $x(t) P_{t} z(t)$ for all $t \geq \max \left\{t^{\prime}, t^{\prime \prime}\right\}$. Hence $x \succ_{R}^{a} z$.

Completeness is trivial.

Next we shall prove variable step anonymity. Let $x \in X^{\infty}$. Let $\pi \in \Pi_{3}$ be associated with the set of steps $\left\{s_{1}, s_{2}, s_{3}, \ldots\right\} \subseteq \mathbb{N}$. Then by anonymity of each $R_{t}$,

$$
x\left(s_{\nu}\right) I_{s_{\nu}} y\left(s_{\nu}\right) \forall s_{\nu} \in\left\{s_{1}, s_{2}, s_{3}, \ldots\right\}
$$

Hence, by definition of the future agreement extension, $x \sim_{R}^{a} y$.

It remains to prove strong Pareto. If $x$ Pareto dominates $y$, then for $s \in \mathbb{N}$ such that $x_{s}>y_{s}$, strong Pareto of each $R_{t}$ implies

$$
x(t) P_{t} y(t) \quad \forall t \geq s
$$

Hence $x \succ_{R}^{a} y$.

Let us prove finite transitivity under the assumption of correlation. Assume that $x \succsim_{R}^{a} y, y \succsim_{R}^{a} z$, and $x[t+1]=y[t+1]=z[t+1]$ for some $t \in \mathbb{N}$. Since $R$ is correlated, Lemma 1 implies that $\succsim_{R}^{a}$ is a strict extension of $R$. Hence, $x(t) R_{t} y(t)$ and $y(t) R_{t} z(t)$, so that $x(t) R_{t} z(t)$ by transitivity of $R_{t}$. Since $\succsim_{R}^{a}$ weakly extends $R$, we have $x=(x(t), x[t+1]) \succsim_{R}^{a}(z(t), x[t+1])=z$.

As mentioned earlier, no binary relation satisfies transitivity, weak Pareto, and variable step anonymity. Therefore, quasi-transitivity and finite transitivity in Lemma 2 cannot be strengthened to transitivity. Fleurbaey and Michel (2003) define $\succsim_{R^{U}}^{a}$ as a variant of the overtaking criterion, and point out that it satisfies quasi-transitivity, completeness, strong Pareto, and variable step anonymity. Lemma 2 generalizes this observation for every binary relation that is the future agreement extension of any sequence of finite generation orderings. 


\subsection{Pairwise continuity}

Continuity means robustness of ranking with respect to small perturbations in data. Though this condition is largely met in the finite dimensional setting, it is quite severe in the setting with an infinite number of generations. It is known that no reasonable finitely anonymous binary relation is continuous with respect to the product (Diamond, 1965), Campbell (Campbell, 1985), or Mackey topology (Shinotsuka, 1998). ${ }^{6}$ We shall introduce a mild continuity condition that is weaker than continuity with respect to these topologies. It is based on the pairwise comparison of utility streams.

Consider any pair $x, y \in X^{\infty}$ and a sequence $\left\{z^{\nu}\right\}_{\nu \in \mathbb{N}}$ in $X^{\infty}$ such that, for some positive integers $s_{1}<s_{2}<s_{3}<\cdots$,

$$
z^{\nu}=\left(x\left(s_{\nu}\right), y\left[s_{\nu}+1\right]\right) \quad \forall \nu \in \mathbb{N} \text {. }
$$

Under various topologies including those aforementioned, the sequence $\left\{z^{\nu}\right\}_{\nu \in \mathbb{N}}$ converges to $y \cdot{ }^{7}$ Hence, whenever

$$
z^{\nu} \succsim y \quad \forall \nu \in \mathbb{N}
$$

continuity with respect to those topologies implies

$$
x \succsim y
$$

We define this weaker notion as a continuity axiom:

Pairwise continuity For every $x, y \in X^{\infty}$, if there exists an infinite set $\mathbb{N}^{\prime} \subseteq \mathbb{N}$ such that $(x(t), y[t+1]) \succsim y$ for all $t \in \mathbb{N}^{\prime}$, then $x \succsim y$.

The first continuity axiom based on the pairwise comparison is Axiom 3 in Brock (1970), which is used in his axiomatization of a Ramsey-Atsumi-von Weizsäcker overtaking criterion (Ramsey, 1928; Atsumi, 1965; von Weizsäcker, 1965). Following Brock's idea, Asheim and Tungodden (2004) and Kamaga and Kojima (2007b) offer interesting variants of his Axiom 3; however, their continuity axioms have no logical relations to continuity with respect to the aforementioned topologies. Conversely, pairwise continuity is implied by continuity with respect to those topologies, and hence any result on this condition has direct logical relevance to Diamond-style impossibility results. Therefore, pairwise continuity derives its idea from both the "topological approach" and the "pairwise approach". This condition will play a crucial role in understanding the future agreement extension. This is the first step:

\footnotetext{
${ }^{6}$ Relations among these and other topologies are summarized by Lauwers (1997a).

${ }^{7}$ In the words of Brown and Lewis (1981), any topology that has some "myopic" feature achieves this convergence. Although the sup topology is a widely studied topology in the literature, it does not have such a feature.
} 
Lemma 3. Let $R=\left\{R_{t}\right\}_{t \in \mathbb{N}}$ be any correlated sequence of finite generation orderings. Then $\succsim_{R}^{a}$ is pairwisely continuous.

Proof. Let $x, y \in X^{\infty}$ be such that, for some infinite set $\mathbb{N}^{\prime} \subseteq \mathbb{N}$,

$$
(x(t), y[t+1]) \succsim_{R}^{a} y \quad \forall t \in \mathbb{N}^{\prime} .
$$

Since $\succsim_{R}^{a}$ is a strict extension of $R$ by Lemma 1 ,

$$
x(t) R_{t} y(t) \quad \forall t \in \mathbb{N}^{\prime} .
$$

Thus by definition of $\succsim_{R}^{a}, x \succsim_{R}^{a} y$.

\section{Main results}

\subsection{Characterizations of the future agreement extension}

The null ordering $\succsim^{n}$ is the binary relation on $X^{\infty}$ defined by $\left[x \succsim^{n} y \forall x, y \in X^{\infty}\right]$, which fails to distinguish utility streams from any normative perspective. We deal with this non-fascinating ordering to make its comparison to the future agreement extension. The next theorem shows that, contrary to the null ordering, the future agreement extension best distinguishes the utility streams among the class of weak extensions satisfying pairwise continuity:

Theorem 1. Let $R=\left\{R_{t}\right\}_{t \in \mathbb{N}}$ be any correlated sequence of finite generation orderings. Then for every binary relation $\succsim$ on $X^{\infty}$ that is a weak extension of $R$ satisfying pairwise continuity,

$$
\succsim_{R}^{a} \subseteq \succsim_{\succsim^{n}}
$$

Furthermore, $\succsim_{R}^{a}$ and $\succsim^{n}$ are weak extensions of $R$ satisfying pairwise continuity.

Proof. Let $\succsim$ be any weak extension of $R$ satisfying pairwise continuity. Let us show that $\succsim_{R}^{a} \subseteq \succsim$. Pick $x, y \in X^{\infty}$ with $x \succsim_{R}^{a} y$. Then there exists an infinite set $\mathbb{N}^{\prime} \subseteq \mathbb{N}$ such that

$$
x(t) R_{t} y(t) \quad \forall t \in \mathbb{N}^{\prime} .
$$

Since $\succsim$ weakly extends $R$,

$$
(x(t), y[t+1]) \succsim(y(t), y[t+1])=y \quad \forall t \in \mathbb{N}^{\prime} .
$$

Thus by pairwise continuity, $x \succsim y$, as desired.

Since $\succsim \subseteq \succsim^{n}$ trivially holds, we have $\succsim_{R}^{a} \subseteq \succsim \subseteq \succsim^{n}$. Lemmas 1 and 3 imply that $\succsim_{R}^{a}$ is a weak extension of $R$ satisfying pairwise continuity. It is obvious that $\succsim^{n}$ is a weak extension of $R$ satisfying pairwise continuity, too. 
Given completeness of $\succsim_{R}^{a}$, the minimality of $\succsim_{R}^{a}$ among the class of binary relations satisfying pairwise continuity implies that $\succsim_{R}^{a}$ is more decisive than any other $\succsim$ belonging to the class. Indeed, for every $x, y \in X^{\infty}, x \sim_{R}^{a} y$ implies $x \sim y$, but $\left[x \succ_{R}^{a} y\right.$ and $\left.x \sim y\right]$ is possible. In the same sense, the maximality of $\succsim^{n}$ implies the least decisiveness of $\succsim^{n}$ among the class. A possible critique to the future agreement extension is that it admits large indifferent classes, but Theorem 1 implies that this extension still keeps the smallest indifferent classes among the class of weak extensions satisfying pairwise continuity.

The next axiom is Asheim and Tungodden's (2004) continuity condition that applies to asymmetric parts of binary relations. ${ }^{8}$

Asheim-Tungodden continuity For every $x, y \in X^{\infty}$, if there exists $s \in \mathbb{N}$ such that $(x(t), y[t+1]) \succ y$ for all $t \geq s$, then $x \succ y$.

Our second main theorem pins down the future agreement extension by strengthening weak extension to strict extension and adding Asheim-Tungodden continuity. ${ }^{9}$

Theorem 2. Let $R=\left\{R_{t}\right\}_{t \in \mathbb{N}}$ be any correlated sequence of finite generation orderings. A strict extension of $R$ satisfies pairwise continuity and Asheim-Tungodden continuity if and only if it is the future agreement extension of $R$.

Proof. Lemmas 1 and 3 imply $\succsim_{R}^{a}$ is a strict extension of $R$ satisfying pairwise continuity. We next show that $\succsim_{R}^{a}$ is Asheim-Tungodden continuous. Let $x, y \in X^{\infty}$ be such that, for some $s \in \mathbb{N}$,

$$
(x(t), y[t+1]) \succ_{R}^{a} y \quad \forall t \geq s .
$$

Since $\succsim_{R}^{a}$ is a strict extension of $R$ by Lemma 1 ,

$$
x(t) \succ_{R}^{a} y(t) \quad \forall t \geq s .
$$

Thus by definition of $\succsim_{R}^{a}, x \succ_{R}^{a} y$, as desired.

\footnotetext{
${ }^{8}$ Asheim and Tungodden call this condition "weak preference continuity".

${ }^{9}$ Both devises are necessary: the null ordering is a weak extension of $R^{U}$ satisfying pairwise continuity and Asheim-Tungodden continuity, but it is not a strict extension of $R^{U}$; the binary relation $\succsim_{R^{U}}^{d}$, which will be defined and studied in Section 4.2, is a strict extension of $R^{U}$ satisfying pairwise continuity but not Asheim-Tungodden continuity. Finally, the following Ramsey-Atsumi-von Weizsäcker overtaking criterion

$$
x \succsim^{O T} y \Longleftrightarrow \exists T, \forall t \geq T, \sum_{s=1}^{t} x_{s} \geq \sum_{s=1}^{t} y_{s}, \quad \forall x, y \in X^{\infty}
$$

is a strict extension of $R^{U}$ satisfying Asheim-Tungodden continuity but not pairwise continuity. These arguments imply the tightness of the conditions in Theorem 2.
} 
Let $\succsim$ be any strict extension of $R$ satisfying pairwise continuity and AsheimTungodden continuity. By Theorem $1, \succsim_{R}^{a} \subseteq \succsim$. To prove $\succsim \subseteq_{\sim}^{a}$, it suffices to show that, for every $x, y \in X^{\infty}$, if $x \succ_{R}^{a} y$, then $x \succ y$. If $x \succ_{R}^{a} y$, then there exists $s \in \mathbb{N}$ such that

$$
x(t) P_{t} y(t) \quad \forall t \geq s .
$$

Since $\succsim$ strictly extends $R,(6)$ implies

$$
(x(t), y[t+1]) \succ(y(t), y[t+1])=y \quad \forall t \geq s .
$$

Hence, by Asheim-Tungodden continuity, $x \succ y$.

\subsection{Future domination extension}

Given a sequence of finite generation orderings $R=\left\{R_{t}\right\}_{t \in \mathbb{N}}$, the future domination extension of $R$ is the binary relation $\succsim_{R}^{d}$ on $X^{\infty}$ defined by, for all $x, y \in X^{\infty}$,

$$
\begin{aligned}
& x \succ_{R}^{d} y \text { if } \exists t \in \mathbb{N}, x(t) P_{t} y(t) \text { and } x[t+1] \geqq y[t+1] \\
& x \sim_{R}^{d} y \text { otherwise. }
\end{aligned}
$$

d'Aspremont (2007) defines an extension of $R$ only by (7). However, then the binary relation so extended fails to be complete. ${ }^{10}$ Adding (8) makes it complete. One may consider that the future domination extension does not generate a well-defined binary relation, since two " $t$ " might exist in (7). However, we do not have to worry about it if $R$ is correlated and strongly Paretian, which is proved in (i) of the next lemma:

Lemma 4. Let $R=\left\{R_{t}\right\}_{t \in \mathbb{N}}$ be any correlated sequence of finite generation orderings satisfying strong Pareto. Then the following statements hold:

(i) $\succsim_{R}^{d}$ is well-defined as a binary relation on $X^{\infty}$;

(ii) $\succsim_{R}^{d}$ is a strict extension of $R$;

(iii) $\succsim_{R}^{d}$ is quasi-transitive, finitely transitive, complete, strongly Paretian, and pairwisely continuous;

(iv) $\succsim_{R}^{a} \subseteq \succsim_{R}^{d}$;

(v) If $R$ is anonymous, then $\succsim_{R}^{d}$ is variable step anonymous.

Proof. See, Appendix.

The next theorem identifies the future agreement extension and the future domination extension as the minimal and maximal strict extensions of strongly Paretian finite generation orderings under quasi-transitivity, strong Pareto, and pairwise continuity:

\footnotetext{
${ }^{10} \mathrm{~d}$ 'Aspremont calls this extension "the simplified criterion".
} 
Theorem 3. Let $R=\left\{R_{t}\right\}_{t \in \mathbb{N}}$ be any correlated sequence of finite generation orderings satisfying strong Pareto. Then for every binary relation $\succsim$ on $X^{\infty}$ that is a strict extension of $R$ satisfying quasi-transitivity, strong Pareto, and pairwise continuity,

$$
\succsim_{R}^{a} \subseteq \succsim \subseteq \succsim_{R}^{d}
$$

Furthermore, $\succsim_{R}^{a}$ and $\succsim_{R}^{d}$ are strict extensions of $R$ satisfying quasi-transitivity, strong Pareto, and pairwise continuity.

Proof. By (i) in Lemma $4, \succsim_{R}^{d}$ is a well-defined complete binary relation. Let $\succsim$ be a strict extension of $R$ satisfying quasi-transitivity, strong Pareto, and pairwise continuity. Theorem 1 implies $\succsim_{R}^{a} \subseteq \succsim$, which in turn implies completeness of $\succsim$.

We shall show $\succsim \subseteq \beth_{R}^{d}$. By completeness of $\succsim$ and $\succsim_{R}^{d}$, it suffices to show that, for every $x, y \in X^{\infty}, x \succ_{R}^{d} y$ implies $x \succ y$. Assume $x \succ_{R}^{d} y$. Then there exists $t \in \mathbb{N}$ such that $x(t) P_{t} y(t)$ and $x[t+1] \geqq y[t+1]$. Consider the case $x[t+1]=y[t+1]$. Then, by strict extension, $x=(x(t), x[t+1]) \succ(y(t), x[t+1])=y$, as desired. Next, consider the case $x[t+1] \geq y[t+1]$. By strong Pareto, $(x(t), x[t+1]) \succ(x(t), y[t+1])$. By strict extension, $(x(t), y[t+1]) \succ(y(t), y[t+1])$. Thus by quasi-transitivity, $x \succ y$, as desired.

The "furthermore part" follows from Lemmas 1-4.

\subsection{Applications}

Using axiomatization results in finite horizon, we can easily characterize extensions of interesting sequences of finite generation orderings. d'Aspremont and Gevers (1977) show that, in the finite horizon setting, the utilitarian ordering is the unique ordering satisfying strong Pareto, anonymity, and an interpersonal comparability condition of utility gains, which is defined in our setting by:

Cardinal unit comparability For every $x, y, x^{\prime}, y^{\prime} \in X^{\infty}$, if there exist a vector $a=\left(a_{1}, a_{2}, \ldots\right)$ and an integer $b>0$ such that

$$
x^{\prime}=\left(a_{1}+b x_{1}, a_{2}+b x_{2}, a_{3}+b x_{3}, \ldots\right) \text { and } y^{\prime}=\left(a_{1}+b y_{1}, a_{2}+b y_{2}, a_{3}+b y_{3}, \ldots\right),
$$

then $x \succsim y \Longleftrightarrow x^{\prime} \succsim y^{\prime}$

Lemma 5. If a binary relation $\succsim$ on $X^{\infty}$ satisfies finite transitivity, completeness, strong Pareto, anonymity, and cardinal unit comparability, then $\succsim i s$ a strict extension of $R^{U}$. 
Proof. Let $z \in X^{\infty}$ and $t \in \mathbb{N}$. It suffices to show that

$$
\sum_{s=1}^{t} x_{s} \geq \sum_{s=1}^{t} y_{s} \Longleftrightarrow(x(t), z[t+1]) \succsim(y(t), z[t+1]) \quad \forall x, y \in X^{\infty}
$$

Define the binary relation $R_{t}^{z}$ on $X^{t}$ by

$$
x(t) R_{t}^{z} y(t) \Longleftrightarrow(x(t), z[t+1]) \succsim(y(t), z[t+1]) \quad \forall x, y \in X^{\infty} .
$$

By the properties of $\succsim, R_{t}^{z}$ so defined is an ordering satisfying strong Pareto, anonymity, and cardinal unit comparability on $X^{t}$. Therefore, by the characterization of the utilitarian ordering by d'Aspremont and Gevers (1977), we have $R_{t}^{z}=R_{t}^{U}$ (this holds independent of the choice of $z$ ). Therefore, (9) holds.

Lemma 5 and our theorems together establish the following characterization of the extensions of the sequence of the utilitarian orderings:

Theorem 4. For every binary relation $\succsim$ on $X^{\infty}$ satisfying finite transitivity, quasitransitivity, completeness, strong Pareto, finite anonymity, cardinal unit comparability, and pairwise continuity,

$$
\succsim_{R^{U}}^{a} \subseteq \succsim \subseteq \succsim_{R^{U}}^{d}
$$

Furthermore, $\succsim_{R^{U}}^{a}$ and $\succsim_{R^{U}}^{d}$ satisfy all these axioms. Whenever $\succsim$ also satisfies AsheimTungodden continuity, $\succsim_{R^{U}}^{a}=\succsim$.

Proof. Given Lemma 5 and Theorems 2 and 3, it remains to prove that $\succsim_{R^{L}}^{a}$ and $\succsim_{R^{L}}^{d}$ satisfy cardinal unit comparability, but we omit its easy proof.

Many studies on utilitarian binary relations on infinite utility streams have been conducted, including Brock (1970), Svensson (1980), Lauwers (1997b,c), Fleurbaey and Michel (2003), Sakai (2003a), Asheim and Tungodden (2004), Basu and Mitra (2007), Banerjee (2006), d'Aspremont (2007), and Kamaga and Kojima (2007a), among others. Roughly speaking, the main difference between these studies and ours is that, they attribute more importance to transitivity than completeness, which we do not. They initially assume transitivity and use Szpilrajn's lemma to obtain complete extensions when necessary ${ }^{11}$, whereas we directly construct complete orderings so as to satisfy quasi-transitivity and finite transitivity. This difference suggests a trade-off between transitivity and constructiveness. However, it should be noted that we are still achieving quasi-transitivity and finite transitivity, which decreases the cost of losing transitivity. Indeed, by paying it, we even succeeded in reconciling with strong

\footnotetext{
${ }^{11}$ See, Svensson (1980) for how to use Szpilrajn's lemma.
} 
Pareto and a strong impartiality requirement of variable step anonymity, which is incompatible with weak Pareto under transitivity (Fleurbaey and Michel, 2003).

The logic used to establish Theorem 4 applies for not only the utilitarian ordering but also for any other ordering. That is, if a set of axioms characterizes strict extensions of some sequence of finite generation orderings as in Lemma 5 and if the binary relation is assumed to be pairwisely continuous, then the same conclusion as that of Theorem 4 holds. This means that our main results can be used as "theorem generators". The following equity axiom is introduced by Hammond (1976):

Hammond equity For every $x, y \in X^{\infty}$, if there exist $t, t^{\prime} \in \mathbb{N}$ such that $x_{s}=y_{s}$ for all $s \neq t, t^{\prime}$ and

$$
y_{t}>x_{t}>x_{t^{\prime}}>y_{t^{\prime}}
$$

then $x \succ y$.

Lemma 6. If a binary relation $\succsim$ on $X^{\infty}$ satisfies finite transitivity, completeness, strong Pareto, anonymity, and Hammond equity, then $\succsim i s$ a strict extension of $R^{L}$.

Proof. Let $z \in X^{\infty}$ and $t \in \mathbb{N}$. Define the binary relation $R_{t}^{z}$ on $X^{t}$ by

$$
x(t) R_{t}^{z} y(t) \Longleftrightarrow(x(t), z[t+1]) \succsim(y(t), z[t+1]) \quad \forall x, y \in X^{\infty} .
$$

By the properties of $\succsim, R_{t}^{z}$ so defined is a transitive, complete, strongly Paretian, anonymous, and Hammond equitable binary relation on $X^{t}$. Therefore, by Hammond's characterization of the leximin ordering ${ }^{12}$, we have $R_{t}^{z}=R_{t}^{L}$ (this holds independent of the choice of $z$ ). Therefore, $\succsim$ strictly extends $R^{L}$.

The next theorem characterizes the extensions of the sequence of the leximin orderings:

Theorem 5. For every binary relation $\succsim$ on $X^{\infty}$ satisfying finite transitivity, quasitransitivity, completeness, strong Pareto, finite anonymity, Hammond equity, and pairwise continuity,

$$
\succsim_{R^{L}}^{a} \subseteq \succsim \subseteq \succsim_{R^{L}}^{d}
$$

Furthermore, $\succsim_{R^{L}}^{a}$ and $\succsim_{R^{L}}^{d}$ satisfy all these axioms. Whenever $\succsim_{\text {also satisfies Asheim- }}$ Tungodden continuity, $\succsim_{R^{L}}^{a}=\succsim$.

Proof. Given Lemma 6 and Theorems 2 and 3, it remains to prove that $\succsim_{R^{L}}^{a}$ and $\succsim_{R^{L}}^{d}$ satisfy Hammond equity, but we omit its easy proof.

\footnotetext{
${ }^{12}$ Hammond's model is more structured, but it is possible to obtain this characterization from his argument. The proof of Proposition 1 in Asheim and Tungodden (2004) contains a direct proof of this fact. They characterize two versions of the leximin ordering in the same model as ours.
} 
We remark that Asheim and Tungodden (2004) and Bossert, Suzumura, and Sprumont (2007) also study intergenerational versions of the leximin ordering; however, as in the case of the utilitarian ordering, no explicit formula is given to satisfy completeness. This is in contrast with $\succsim_{R^{L}}^{a}$ and $\succsim_{R^{L}}^{d}$.

\section{Concluding comments}

We introduced the notions of weak extension, strict extension, and correlated sequences to systematically analyze how to construct welfare criteria on infinite utility streams using welfare criteria on finite dimensional spaces. We then proposed an explicit method for constructing intergenerational welfare criteria, the future agreement extension. This method always achieves quasi-transitivity, finite transitivity, completeness, and pairwise continuity as well as all standard normative requirements including strong Pareto and a strong impartiality requirement of variable step anonymity. The cost of obtaining these desirable properties is the violation of transitivity, but it was an inevitable consequence from the non-existence of transitive, weakly Paretian, and variable step anonymous binary relations. However, since we still successfully achieve quasi-transitivity and finite transitivity, our arguments suggest that the relaxation of transitivity is an attractive way to obtain reasonable intergenerational welfare criteria, given indeed many non-existence and non-constructive existence results involving transitivity. This approach is in line with the study by Sen (1970), who considers a departure from Arrow's impossibility theorem by relaxing transitivity of social preferences to quasi-transitivity in his famous Pareto-extension theorem. Our study also highlights the possibility of respecting variable step anonymity, which has so far received little attention.

We are not insisting that the future agreement extension is a perfect way of extension, since it admits large indifference classes as the cost of completeness. However, as Theorem 1 shows, this is the most decisive way under weak extension and pairwise continuity. We consider that, given many existing impossibility results in the literature, this extension is an acceptable compromise to obtain positive results as much as possible. In fact, we found its compatibility with various desirable properties including strong Pareto, variable step anonymity, or Hammond equity. We hope that this study motivates the further future research that contributes to other explicit ways of extending finite dimensional welfare criteria. 


\section{Appendix}

Proof of Lemma 4. The proof makes use of the following fact:

Fact. For every $x, y \in X^{\infty}$, if $x \succ_{R}^{d} y$, then there exists $s \in \mathbb{N}$ such that for every $\boldsymbol{t} \geq \boldsymbol{s}, \boldsymbol{x}(\boldsymbol{t}) \boldsymbol{P}_{\boldsymbol{t}} \boldsymbol{y}(\boldsymbol{t})$ : Let $x, y \in X^{\infty}$ be such that $x \succ_{R}^{d} y$. Then there exists $s \in \mathbb{N}$ for which $x(s) P_{s} y(s)$ and $x[s+1] \geqq y[s+1]$. By correlation of $R$,

$$
(x(s), y[s+1, t]) P_{t}(y(s), y[s+1, t]) \quad \forall t \geq s+1 .
$$

For every $t \geq s+1$ such that $x[s+1, t]=y[s+1, t]$, reflexivity of $R_{t}$ implies

$$
(x(s), x[s+1, t]) I_{t}(x(s), y[s+1, t]) .
$$

For every $t \geq s+1$ such that $x[s+1, t] \geq y[s+1, t]$, strong Pareto of $R_{t}$ implies

$$
(x(s), x[s+1, t]) P_{t}(x(s), y[s+1, t]) .
$$

In both cases, (10) and transitivity of $R_{t}$ imply

$$
x(t)=(x(s), x[s+1, t]) P_{t}(y(s), y[s+1, t])=y(t) \quad \forall t \geq s+1,
$$

as desired.

(i) Let us show that $\succsim$ is well-defined as a binary relation. It suffices to prove that there exist no $x, y \in X^{\infty}$ such that $x \succ_{R}^{d} y$ and $y \succ_{R}^{d} x$. If such $x, y$ exist, then Fact implies that for any large $t, x(t) P_{t} y(t)$ and $y(t) P_{t} x(t)$, a contradiction.

(ii) We shall prove that $\succsim_{R}^{d}$ strictly extends $R$. Let $x, y, z \in X^{\infty}$ and $T \in \mathbb{N}$. If $x(T) P_{T} y(T)$, then by definition of $\succsim_{R}^{d}$,

$$
(x(T), z[T+1]) \succ_{R}^{d}(y(T), z[T+1]),
$$

as desired.

Next, assume $x(T) I_{T} y(T)$. Then by correlation of $R$,

$$
(x(T), z[T+1, t]) I_{t}(y(T), z[T+1, t]) \quad \forall t \geq T .
$$

Let $v \equiv(x(T), z[T+1])$ and $w \equiv(y(T), z[T+1])$. To prove $v \sim_{R}^{d} w$, suppose, by contradiction, that $v \succ_{R}^{d} w$. Then there exists $S \in \mathbb{N}$ such that

$$
v(S) P_{S} w(S) \text { and } v[S+1] \geqq w[S+1] .
$$

Therefore, by strong Pareto, correlation, and transitivity,

$$
(v(S), v[S+1, s]) R_{s}(v(S), w[S+1, s]) P_{s}(w(S), w[S+1, s]) \quad \forall s \geq S,
$$


where $(v(S), z[S+1, s]) I_{s}(v(S), w[S+1, s])$ holds if and only if $z[S+1, s]=w[S+1, s]$. By definitions of $v, w$ and (12),

$$
(x(T), z[T+1, t]) P_{t}(y(T), z[T+1, t]) \quad \forall t \geq \max \{T, S\}
$$

which contradicts (11).

(iii) Completeness and strong Pareto are trivial. Let us prove quasi-transitivity. Assume $x \succ_{R}^{d} y$ and $y \succ_{R}^{d} z$. Then, by Fact, there exists a large $t^{\prime} \in \mathbb{N}$ such that

$$
\begin{aligned}
& x(t) P_{t} y(t) \quad \forall t \geq t^{\prime}, \\
& y(t) P_{t} z(t) \quad \forall t \geq t^{\prime},
\end{aligned}
$$

and then transitivity of $R_{t}$ implies

$$
x(t) P_{t} z(t) \quad \forall t \geq t^{\prime}
$$

Since $x \succ_{R}^{d} y$ and $y \succ_{R}^{d} z$, by definition of $\succsim_{R}^{d}$, there exists a large $t^{\prime \prime} \in \mathbb{N}$ such that

$$
x\left[t^{\prime \prime}+1\right] \geqq y\left[t^{\prime \prime}+1\right] \geqq z\left[t^{\prime \prime}+1\right] \quad \forall t \geq t^{\prime \prime}
$$

Let $s \equiv\left\{t^{\prime}, t^{\prime \prime}\right\}$. Then by $(13,14)$,

$$
x(s) P_{s} z(s) \text { and } x[s+1] \geqq z[s+1],
$$

and hence $x \succ_{R}^{d} z$ by definition of $\succsim_{R}^{d}$.

Let us prove finite transitivity. Let $x, y, z \in X^{\infty}$ and $t \in \mathbb{N}$ be such that $x \succsim_{R}^{d} y$, $y \succsim_{R}^{d} z$, and $x[t+1]=y[t+1]=z[t+1]$. Since $\succsim$ strictly extends $R$,

$$
x(s) R_{s} y(s) \text { and } y(s) R_{s} z(s) \quad \forall s \geq t,
$$

so by transitivity of $R_{s}$,

$$
x(s) R_{s} z(s) \quad \forall s \geq t
$$

By Fact, $z \succ_{R}^{d} x$ contradicts (15), and hence by completeness, $x \succsim_{R}^{d} z$.

We shall next prove pairwise continuity. Let $x, y \in X^{\infty}$ be such that, for some infinite set $\mathbb{N}^{\prime} \subseteq \mathbb{N}$

$$
(x(t), y[t+1]) \succsim_{R}^{d} y \quad \forall t \in \mathbb{N}^{\prime} .
$$

Since $\succsim_{R}^{d}$ strictly extends $R$,

$$
x(t) R_{t} y(t) \quad \forall t \in \mathbb{N}^{\prime} .
$$


If $y \succ_{R}^{d} x$, then Fact implies the existence of $s \in \mathbb{N}$ such that

$$
y(t) P_{t} x(t) \forall t \geq s
$$

which contradicts (16). Therefore, by completeness, $x \succsim_{R}^{d} y$.

(iv) To prove $\succsim_{R}^{a} \subseteq \succsim_{R}^{d}$, we use a contraposition argument. If $y \succ_{R}^{d} x$, then by Fact, there exists $s \in \mathbb{N}$ such that for every $t \geq s, y(t) P_{t} x(t)$. This immediately implies $y \succ_{R}^{a} x$.

(v) We omit the easy proof.

\section{References}

Asheim, G.B. and B. Tungodden (2004) "Resolving distributional conflicts between generations," Economic Theory, Vol. 24, pp. 221-230.

Atsumi, H. (1965) "Neoclassical growth and the efficient program of capital accumulation," Review of Economic Studies, Vol. 32, pp. 127-136.

Basu, K. and T. Mitra (2007) "Utilitarianism for infinite utility streams: A new welfare criterion and its axiomatic characterization," Journal of Economic Theory, Vol. 133, pp. $350-373$.

Banerjee, K. (2006) "On the extension of the utilitarian and Suppes-Sen social welfare relations to infinite utility streams," Social Choice and Welfare, Vol. 27, pp. 327-339.

Bossert, W., Y. Sprumont, and K. Suzumura (2007) "Ordering infinite utility streams," Journal of Economic Theory, Vol. 135, pp. 579-589.

Brock, W.A. (1970) "An axiomatic basis for the Ramsey-Weizsacker overtaking criterion," Econometrica, Vol. 38, pp. 927-929.

Brown, D.J. and L.M. Lewis (1981) "Myopic economic agents," Econometrica, Vol. 49, pp. 359-368.

Campbell, D. (1985) "Impossibility theorems and infinite horizon planning," Social Choice and Welfare, Vol. 2, pp. 283-293.

Chambers, C.P. (2008) "Intergenerational equity: sup, inf, lim sup, and lim inf," forthcoming in Social Choice and Welfare.

d'Aspremont, C. (2007) "Formal welfarism and intergenerational equity," in Intergenerational Equity and Sustainability, eds. by J. Roemer and K. Suzumura. Palgrave. 
d'Aspremont, C. and L. Gevers (1977) "Equity and the informational basis of collective choice," Review of Economic Studies, Vol. 46, pp. 199-210.

d'Aspremont, C. and L. Gevers (2002) "Social welfare functionals and interpersonal comparability," in Handbook of Social Choice of Welfare (Ch. 10), eds. by K.J. Arrow, A.K. Sen, and K. Suzumura. North-Holland.

Diamond, P. (1965) "The evaluation of infinite utility streams," Econometrica, Vol. 33, pp. 170-177.

Fleurbaey, M. and P. Michel (2001) "Transfer principles and inequality aversion, with an application to optimal growth," Mathematical Social Sciences, Vol. 42, pp. 1-11.

Fleurbaey, M. and P. Michel (2003) "Intertemporal equity and the extension of the Ramsey criterion," Journal of Mathematical Economics, Vol. 39, pp. 777-802.

Hammond, P.J. (1976) "Equity, Arrow's conditions, and Rawls' difference principle," Econometrica, Vol. 44, pp. 793-804.

Hara, C., T. Shinotsuka, K. Suzumura, and Y. Xu (2008) "Continuity and egalitarianism in the evaluation of infinite utility streams," Social Choice and Welfare, Vol. 31, pp. 179-191.

Kamaga, K. (2008) "Extended anonymity and continuous ethical preferences on infinite utility streams," mimeo, Waseda University.

Kamaga, K. and T. Kojima (2007a) "On the leximin and utilitarian overtaking criteria with extended anonymity," mimeo, Waseda University.

Kamaga, K. and T. Kojima (2007b) "Q-anonymous social welfare relations on infinite utility streams," mimeo, Waseda University.

Lauwers, L. (1997a) "Continuity and equity with infinite horizons," Social Choice and Welfare, Vol. 14, pp. 345-356.

Lauwers, L. (1997b) "Infinite utility: insisting on strong monotonicity," Australasian Journal of Philosophy, Vol. 75, pp. 222-233.

Lauwers, L. (1997c) "Rawlsian equity and generalised utilitarianism with an infinite population," Economic Theory, Vol. 9, pp. 143-150.

Lauwers, L. (2007) "Ordering infinite utility streams comes at the cost of a non-Ramsey set," mimeo, K.U. Leuven. 
Mitra, T. and K. Basu (2007) "On the existence of Paretian social welfare relations for infinite utility streams with extended anonymity," in Intergenerational Equity and Sustainability, eds. by J. Roemer and K. Suzumura. Palgrave.

Sakai, T. (2003a) "An axiomatic approach to intergenerational equity," Social Choice and Welfare, Vol. 20, pp. 167-176.

Sakai, T. (2003b) "Intergenerational preferences and sensitivity to the present," Economic Bulletin, Vol. 4, pp. 1-5.

Sakai, T. (2006) "Equitable intergenerational preferences on restricted domains," Social Choice and Welfare, Vol. 27, pp. 41-54.

Sen, A.K. (1970) Collective Choice and Social Welfare, Holden Day.

Shinotsuka, T. (1998) "Equity, continuity, and myopia: a generalization of Diamond's impossibility theorem," Social Choice and Welfare, Vol. 15, pp. 21-30.

Svensson, L.-G. (1980) "Equity among generations," Econometrica, Vol. 48, pp. 1951-1956.

Szpilrajn, E. (1930) "Sur l' extension de l' ordre partiel," Fundamenta Mathematicae, Vol. 16, pp. 386-389.

van Liedekerke, L. and L. Lauwers (1997) "Sacrificing the patrol: utilitarianism, future generations, and infinity," Economics and Philosophy, Vol. 13, pp. 159-174.

von Weizsäcker, C.C. (1960) "Existence of optimal programmes of accumulation for an infinite time horizon," Review of Economic Studies, Vol. 32, pp. 85-104.

Zame, W.R. (2007) "Can utilitarianism be operationalized?," Theoretical Economics, Vol. 2, pp. $187-202$. 